\title{
Overexpression of $\mathrm{CD64}$ on $\mathrm{CD}^{++} 4^{+\mathrm{CD}^{-}}{ }^{-}$and $\mathrm{CD}^{++}{ }^{++} \mathrm{CD}^{+}{ }^{+}$monocytes of rheumatoid arthritis patients correlates with disease activity
}

\author{
QING LUO ${ }^{1 *}$, PENGCHENG XIAO $^{2}, \mathrm{XUE} \mathrm{LI}^{3}, \mathrm{ZHEN} \mathrm{DENG}^{3}, \mathrm{CHENG} \mathrm{QING}^{4}, \mathrm{RIGU} \mathrm{SU}^{3}$, \\ JIANQING XU ${ }^{3}$, YANG GUO ${ }^{1}$, ZIKUN HUANG ${ }^{1}$ and JUNMING LI ${ }^{1}$
}

${ }^{1}$ Department of Clinical Laboratory, The First Affiliated Hospital of Nanchang University, Nanchang, Jiangxi 330006;
${ }^{2}$ Department of Clinical Center Laboratory, Zhuzhou Central Hospital, Zhuzhou, Hunan $412007 ;{ }^{3}$ Department of
Medical College, Nanchang University; ${ }^{4}$ Department of Intensive Care Unit, The First Affiliated Hospital of
Nanchang University, Nanchang, Jiangxi 330006, P.R. China

Received October 25, 2017; Accepted June 26, 2018

DOI: $10.3892 /$ etm.2018.6452

\begin{abstract}
It is well-known that monocytes are a heterogeneous cell population and different monocyte subsets play important roles in rheumatoid arthritis (RA). Cluster of differentiation (CD)64 is one of Fc receptor, which initiates immunological and inflammatory reactions. However, the roles in RA remain to be elucidated. In the present study, the expression of CD64, CD40, CD163, CD206, HLA-DR, CD80 and CD86 on monocytes and the expression of CD64 on monocyte subsets were determined by flow cytometry. The expression of CD64 on monocyte subsets in patients with RA was further analyzed for their correlation with markers of autoimmune response, inflammation, disease activity of RA and serum cytokines. Compared to the health volunteers, the expression of CD64 on monocytes and each monocyte subset were significantly elevated in RA patients. The expression of CD64 on $\mathrm{CD} 14^{++} \mathrm{CD} 16^{-}$and $\mathrm{CD} 14^{++} \mathrm{CD} 16^{+}$ monocytes were positively correlated with erythrocyte sedimentation rate (ESR), C-reactive protein (CRP), rheumatoid factor (RF), anti-citrullinated protein antibodies (ACPA) and disease activity score 28 (DAS28). Furthermore, the expression of CD64 on $\mathrm{CD}_{14}{ }^{++} \mathrm{CD} 16^{+}$monocytes was found to be associated with the serum level of IL-6. In conclusions, these data demonstrated the expression of CD64 on $\mathrm{CD} 14^{++} \mathrm{CD} 16$ and $\mathrm{CD} 14^{++} \mathrm{CD} 16^{+}$monocytes are elevated and associated with the disease activity in RA.
\end{abstract}

Correspondence to: Dr Junming Li or Dr Zikun Huang, Department of Clinical Laboratory, The First Affiliated Hospital of Nanchang University, 17 Yongwai Zheng Jie Road, Nanchang, Jiangxi 330006, P.R. China

E-mail: lisir361@163.com

E-mail: 491353062@qq.com

${ }^{*}$ Contributed equally

Key words: rheumatoid arthritis, CD64, monocyte subsets

\section{Introduction}

Rheumatoid arthritis (RA) is a chronic inflammatory disease which causes pain and dysfunction and leads to the destruction of joints. Activation and recruitment of immune cells, especially lymphocytes and monocytes into the joints, are major characters of RA $(1,2)$. The mechanisms underlying RA are complex, including genetic and environmental factors, as well as abnormalities of both innate immunity and adaptive immunity (3). Although the etiopathology of RA is not fully understood, it is known that monocytes/macrophages, neutrophils, $\mathrm{T}$ cells and $\mathrm{B}$ cells are involved in the mechanisms that drive the onset of RA (4). These cells play a key role in the progression of RA through the production of proinflammatory cytokines, leading to the development of an inflammatory environment and immune cell recruitment in the joints.

In humans, monocytes are a heterogeneous cell population composed of three distinct subsets based on their expression of CD14 and CD16 (5). The CD14 ${ }^{++} \mathrm{CD} 16$ classical subset is the most prominent of all circulating monocytes. The second monocyte subset expresses levels of both CD14 and CD16 $\left(\mathrm{CD} 14^{++} \mathrm{CD} 6^{+}\right)$. It is referred to as intermediate monocytes. The third subset comprises nonclassical monocytes that express low levels of CD14 and high levels of CD16 $\left(\mathrm{CD} 14^{+} \mathrm{CD} 16^{++}\right)$. The $\mathrm{CD} 14^{++} \mathrm{CD} 16^{-}$monocyte is the major subset, while the $\mathrm{CD}^{++}{ }^{++} \mathrm{CD}_{16}{ }^{+}$and $\mathrm{CD} 14^{+} \mathrm{CD} 16^{++}$subsets occur in lower numbers than $\mathrm{CD}_{14}{ }^{++} \mathrm{CD} 16^{-}$monocyte (6). The two $\mathrm{CD} 14^{++}$ subsets are thus recognized to expand in various inflammatory diseases and are suggested to play a significant role in disease processes $(7,8)$. Recent reports have shown that the proportion of monocyte subsets was aberrant in RA patients $(9,10)$.

CD64 (FcgRI), a Fc receptor for $\mathrm{IgG}$, is constitutively expressed on macrophages and monocytes. CD64 is the high-affinity receptor for monomeric IgG or Ig in immune complexes that can initiates immunological and inflammatory reactions on immune competent cells, including monocytes and joint-stationed macrophages (11-13). Evidences from both human studies and animal models have demonstrated 
that CD64 play a important role in RA pathogenesis $(14,15)$. However, previous monocyte CD64 expression studies in RA have reported conflicting findings, showing increased, decreased or similar expressions compared with health volunteers (HV) (16-18). The role of CD64 on monocytes in the pathogenesis of RA remains to be clarified. And, whether CD64 can regulate the function of monocyte subsets in RA remains to be clarified.

In the present study, we detected the expression of CD64 on monocyte subsets in patients with RA and HV. The correlation between the expression of CD64 on monocyte subsets and the activity of RA was also investigated. Moreover, the cytokines secretion of $\mathrm{CD}^{+} 4^{+}$monocyte subsets in patients with RA was measured.

\section{Patients and methods}

Subjects. A total of 46 patients fulfilled the revised American College of Rheumatology criteria for RA (19) were recruited from the First Affiliated Hospital of Nanchang University. Among them, 5 patients were new-onset RA ( $<6$ months disease duration) (20). All patients were administered disease-modifying anti-rheumatic drugs (DMARDs), including glucocorticoid and immunosuppressor therapy. Disease activity of RA was calculated using the disease activity score 28 (DAS28) (21). The patient characteristics of this group are shown in Table I. In addition, the present study included $22 \mathrm{HV}$ (female $81.8 \%$, mean age $51.2 \pm 11.6$ years) who were unrelated to the patients and did not have inflammatory or autoimmune diseases. The study was approved by the Ethics Committee of the First Affiliated Hospital of Nanchang University (019) and was carried out in compliance with the Helsinki Declaration. Written informed consent was obtained from all participants before they entered the present study.

Flow cytometry analysis. Peripheral blood mononuclear cells (PBMCs) were isolated from the fresh peripheral blood of RA patients and HV on Ficoll-Paque gradient (Sigma-Aldrich; Merck KGaA, Darmstadt,Germany). The membrane molecules of monocytes were analyzed immediately using flow cytometry. The following antibodies were used: ECD-conjugated anti-CD14, PC5-conjugated anti-CD16 (BD Biosciences, San Diego CA, USA), PE-conjugated anti-CD163, anti-CD206, and anti-CD86, FITC-conjugated anti-CD80, anti-CD40, anti-CD64, anti-HLA-DR (MIH clones; eBioscience; Thermo Fisher Scientific, Inc., Waltham, MA, USA). Monocyte subsets identified as detailed above based on their expression of CD14 and CD16 (5). Briefly, 5x10 ${ }^{5}$ PBMCs was incubated simultaneously with $10 \mu \mathrm{l}$ of ECD-conjugated anti-CD14, $10 \mu 1$ of PC5-conjugated anti-CD16, and PE-conjugated anti-CD64 on ice in the dark for $30 \mathrm{~min}$. Cells incubated with PE-conjugated mouse $\mathrm{IgG}$ were used as isotype controls. Expression of CD64 was analyzed on each monocyte subset using a CYTOMICS FC 500 flow cytometer (Beckman Coulter, Inc., Brea, CA, USA) and data analyzed with the associated software programs (CXP).

Serum CRP, IgG, C3 and C4 measurement. The concentrations of serum C-reactive Protein (CRP), Immunoglobulin G (IgG), Complement $3(\mathrm{C} 3)$ and Complement 4 (C4) were determined by nephelometry methods according to the instructions described by the manufacturer (IMMUNE800; Beckman Coulter, Inc.).

Erythrocyte sedimentation rate (ESR) blood routine measurement. ESR and blood routine were determined according to the instructions described by the manufacturer.

Autoantibody measurement. Level of rheumatoid factor (RF) was determined using nephelometry methods according to the instructions described by the manufacturer (IMMUNE800; Beckman Coulter, Inc.). Anti-citrullinated protein antibodies (ACPA) from serum $\mathrm{IgG}$ were measured using commercially ELISA kits (Kexin, Shanghai, China).

Cytokine measurement. Human IL-10,IL-6 and IL-8 (Signalway Antibody LLC, College Park, MD, USA) were measured using commercially available enzyme-linked immunosorbent assays according to the manufacturers' instructions.

Statistical analysis. Statistical analysis and graphic presentation were carried out with GraphPad Prism v.5.0 (GraphPad Software, Inc., La Jolla, CA, USA). In addition, Student's t-test was used where the normality test passed; otherwise, the nonparametric Mann-Whitney test was used to analyze the data. Likewise, the Pearson method or the nonparametric Spearman method was used for correlation analysis. $\mathrm{P}<0.05$ was considered to indicate a statistically significant difference.

\section{Result}

Increased expression of CD64 in the monocytes of RA patients. The monocytes in PBMCs were analyzed for the expression of membrane molecules including CD40, CD64, CD163, CD206, HLA-DR, CD80 and CD86 by flow cytometry. Representative dot plots of population gating and CD64 expressing cells from RA patients and HV were showed in Fig. 1A. Results showed that the expression of CD64 on monocytes was significantly elevated in RA patients compared to $\mathrm{HV}$ ( $\mathrm{P}=0.0103$; Fig. 1B). No significant difference was observed in the expression of CD40, CD163, CD206, HLA-DR, CD80, CD86 on monocytes between RA patients and HV (Fig. 1).

Proportions of each monocyte subset. Representative dot plots of each monocyte subset from flow cytometry analysis of $\mathrm{CD}_{14}^{++}{ }^{+} \mathrm{CD} 16^{-}$(P1), CD14 ${ }^{++} \mathrm{CD}^{+} 6^{+}$(P2) and $\mathrm{CD} 14^{+}$ $\mathrm{CD}^{++}$(P3) blood monocytes from HV and RA patients are shown in Fig. 2A. The three monocyte subsets in total monocytes of peripheral blood cells from patients with RA and healthy volunteers are shown in Fig. 2B. The proportion of $\mathrm{CD} 14^{++} \mathrm{CD} 16^{+}$monocytes in patients with RA was significantly higher than that in $\mathrm{HV}(\mathrm{P}<0.0001)$, while the proportion of $\mathrm{CD} 14^{++} \mathrm{CD} 16{ }^{-}$and $\mathrm{CD} 14^{+} \mathrm{CD} 16^{++}$monocytes in patients with RA was significantly lower than that in $\mathrm{HV}(\mathrm{P}=0.0237$; $\mathrm{P}=0.0044$ ). Moreover, as showed in Fig. 2C, the proportion of $\mathrm{CD} 14^{++} \mathrm{CD} 16^{+}$monocytes in PBMCs was significantly increased in patients with RA than that in $\mathrm{HV}(\mathrm{P}=0.0011)$, when that of $\mathrm{CD} 14^{++} \mathrm{CD} 16$ and $\mathrm{CD} 14^{+} \mathrm{CD} 16^{++}$monocytes did not differ between the two groups. 
Table I. Clinical characteristics of patients with RA and HV.

\begin{tabular}{lcc}
\hline Categories & RA $(\mathrm{n}=46)$ & $\mathrm{HV}(\mathrm{n}=22)$ \\
\hline Females, n (\%) & $38(82.6)$ & $19(81.8)$ \\
Age, mean (SD), years & $57.3 \pm 12.0$ & $51.2 \pm 11.6$ \\
DAS28, mean (SD) & $4.4 \pm 1.9$ & - \\
ACPA (+, >25 RU/ml), n (\%) (33 patients) & $25(75.7)$ & - \\
RF (+, >20 IU/ml), n (\%) (33 patients) & $22(66.7)$ & - \\
C3, mean (SD), g/l (30 patients) & $0.9 \pm 0.2$ & - \\
C4, mean (SD), g/l (30 patients) & $0.2 \pm 0.06$ & - \\
IgG, mean (SD), g/l (30 patients) & $12.9 \pm 6.1$ & - \\
WBC, mean (SD) & $6.9 \pm 3.0$ & - \\
Neutrophil count, mean (SD) & $4.8 \pm 2.5$ & - \\
The percent of neutrophil, mean (SD), $\%$ & $68.0 \pm 8.9$ & - \\
ESR, mean (SD), mm/h & $47.2 \pm 34.6$ & - \\
CRP, mean (SD), mg/l (37 patients) & $19.7 \pm 22.4$ & - \\
\hline
\end{tabular}

RA, rheumatoid arthritis; HV, health volunteers; ACPA, anti-citrullinated protein antibodies; C3, complement 3; C4, complement 4; DAS28, disease activity score 28; ESR, erythrocyte sedimentation rate; IgG, immunoglobulin G; RF, rheumatoid factor; WBC, white blood cell; SD, standard deviation.

A


B
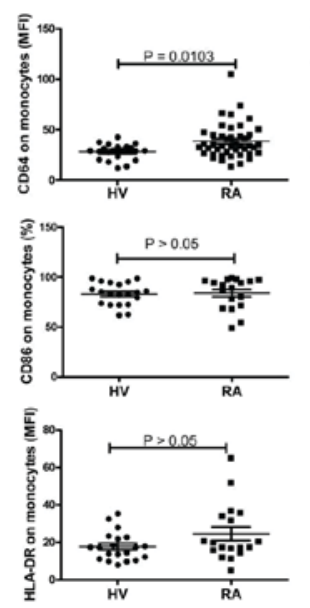
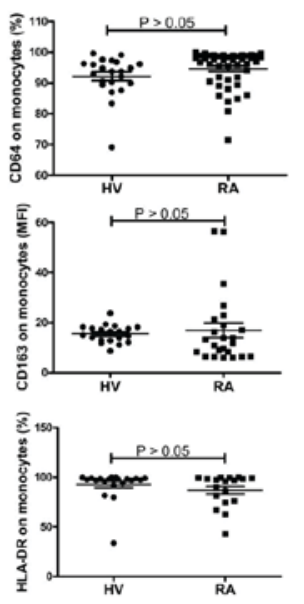
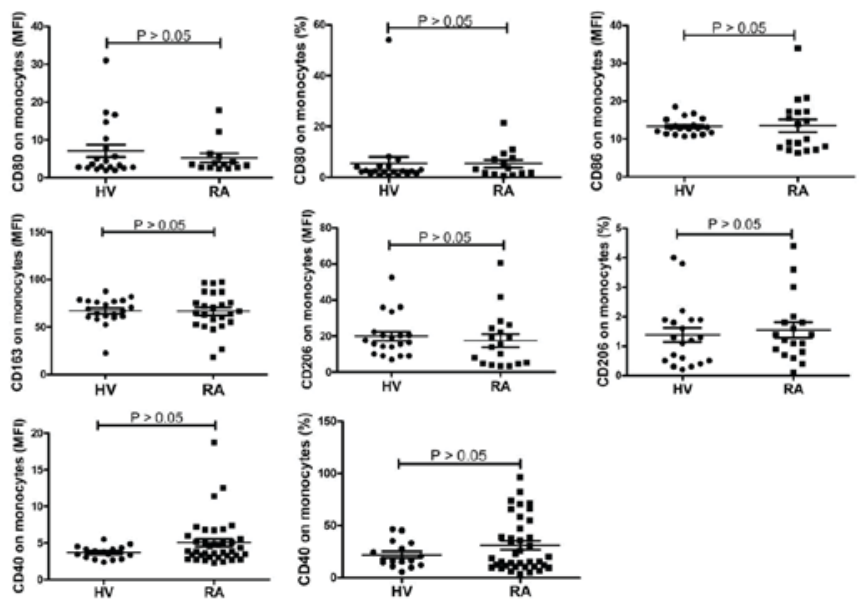

Figure 1. CD40, CD64, CD163, CD206, HLA-DR, CD80 and CD86 expression on monocytes. (A) Representative dot plots of population gating and CD64 expressing cells from RA patients and HV. (B) Summary data of the expression of CD40, CD64, CD163, CD206, HLA-DR, CD80 and CD86 on monocytes. RA, rheumatoid arthritis; HV, health volunteers.

CD64 expression on monocyte subsets in RA patients and $H V$. To determine the expression profile of CD64 on monocyte subsets in RA patients and $\mathrm{HV}$, we used flow cytometry to assess the expression of CD64 on monocyte subsets including $\mathrm{CD} 14^{++} \mathrm{CD} 16^{-}$monocytes, CD $14^{++} \mathrm{CD} 16^{+}$ monocytes, and $\mathrm{CD} 14^{+} \mathrm{CD} 16^{++}$monocytes (Fig. 3). Data showed that although the frequency of CD64-expressing
CD14 ${ }^{++} \mathrm{CD} 16-$ monocytes, CD64-expressing $\mathrm{CD}^{-} 4^{++} \mathrm{CD} 16^{+}$ monocytes, and CD64-expressing $\mathrm{CD} 14^{+} \mathrm{CD} 16^{++}$monocytes did not differ between the two groups (Fig. 3B), the mean fluorescence intensity (MFI) of CD64 on CD14 ${ }^{++} \mathrm{CD} 166^{-}$monocytes, $\mathrm{CD} 14^{++} \mathrm{CD} 16^{+}$monocytes and $\mathrm{CD} 14^{+} \mathrm{CD} 16^{++}$monocytes were significantly elevated in patients with RA compared to HV $(\mathrm{P}<0.0001$; Fig. 3C). Further, results showed that the 

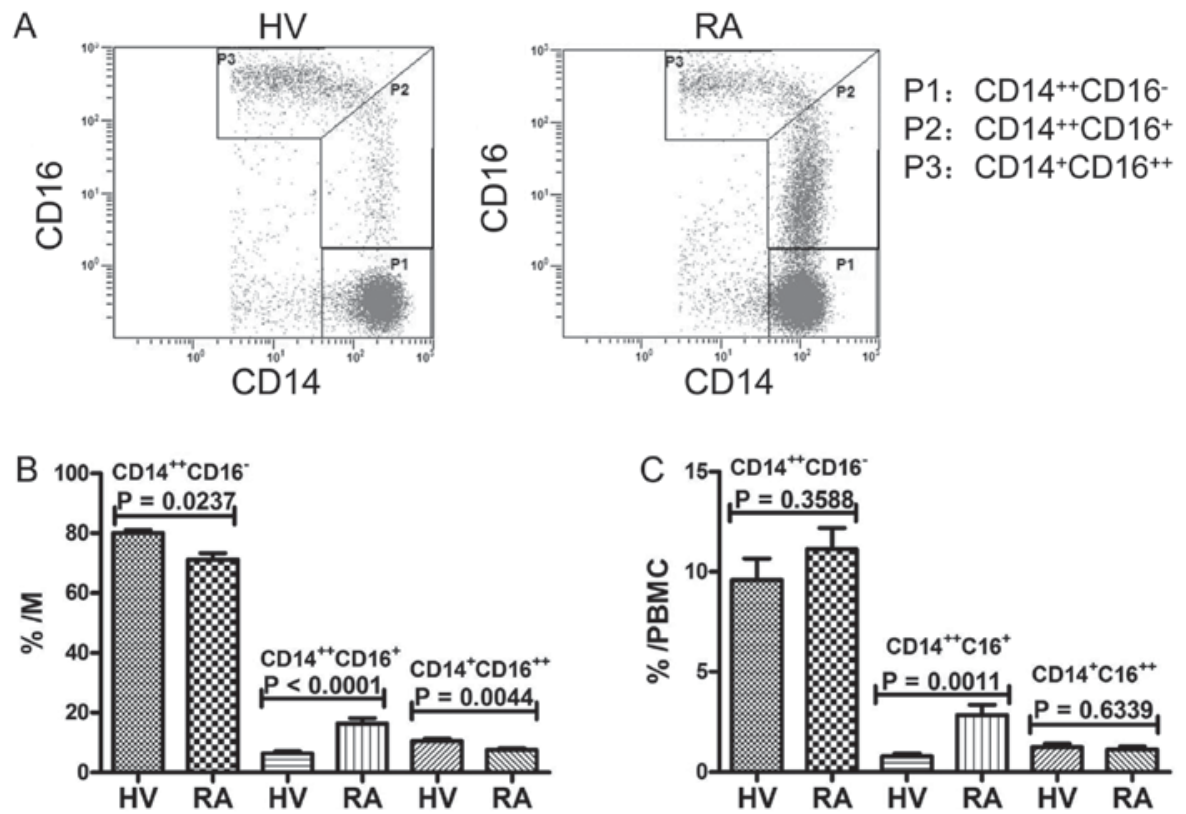

Figure 2. Levels of monocyte subsets in RA patients. (A) Flow cytometry analysis of CD14 ${ }^{++} \mathrm{CD} 16-(\mathrm{P} 1), \mathrm{CD} 14^{++} \mathrm{CD} 16^{+}(\mathrm{P} 2)$ and $\mathrm{CD} 14^{+} \mathrm{CD} 16^{++}(\mathrm{P} 3)$ blood monocytes from HV and RA patients. Data are cell populations of representative donors. (B) Percentage of blood monocyte subsets in monocytes from HV and RA patients. (C) Percentage of blood monocyte subsets in PBMCs from HV and RA patients. RA, rheumatoid arthritis; HV, health volunteers; PBMCs, peripheral blood mononuclear cells.

frequency of CD64-expressing CD14++ $\mathrm{CD} 16^{-}$monocytes and CD64-expressing $\mathrm{CD} 14^{++} \mathrm{CD} 16^{+}$monocytes were significantly elevated compared to CD64-expressing $\mathrm{CD} 14^{+} \mathrm{CD} 16^{++}$ monocytes in both HV $(\mathrm{P}<0.0001$; Fig. 3D) and RA patients $(\mathrm{P}<0.0001$; Fig. 3F). And, the frequency of CD64-expressing $\mathrm{CD} 14^{++} \mathrm{CD} 16^{-}$monocytes was significantly elevated compared to CD64-expressing $\mathrm{CD} 14^{++} \mathrm{CD} 6^{+}$monocytes in RA patients $(\mathrm{P}<0.0001$; Fig. 3F), but no differences was found in HV $(\mathrm{P}=0.1389$; Fig. 3D). As showed in Fig. 3E and G, the expression of CD64 on $\mathrm{CD}_{14}{ }^{++} \mathrm{CD} 16$ monocytes and $\mathrm{CD} 14^{++} \mathrm{CD} 16^{+}$monocytes were significantly elevated compared to $\mathrm{CD} 14^{+} \mathrm{CD} 16^{++}$ monocytes in both RA patients $(\mathrm{P}<0.0001)$ and $\mathrm{HV}(\mathrm{P}<0.0001)$. Moreover, we investigated the correlation between the expression of CD64 on monocyte subsets and the proportions of each monocyte subset. Data showed that the proportion of $\mathrm{CD} 14^{++} \mathrm{CD} 16^{-}$monocytes negatively correlated with the expression of $\mathrm{CD} 64$ on $\mathrm{CD} 14^{++} \mathrm{CD} 16^{-}$monocytes ( $\mathrm{r}=0.4541$, $\mathrm{P}=0.0002$; Fig. $3 \mathrm{H}$ ), whereas the proportion of $\mathrm{CD} 14^{++} \mathrm{CD} 16^{+}$ monocytes positively correlated with the expression of CD64 on $\mathrm{CD}_{14}{ }^{++} \mathrm{CD}_{16} 6^{+}$monocytes in RA patients $(\mathrm{r}=0.4352, \mathrm{P}=0.0032$; Fig. 3I). But no obvious correlation was observed between the expression of $\mathrm{CD} 64$ on $\mathrm{CD} 14^{+} \mathrm{CD} 16^{++}$monocytes and the proportions of $\mathrm{CD} 14^{+} \mathrm{CD} 16^{++}$monocytes $(\mathrm{r}=0.1910, \mathrm{P}=0.2140$; Fig. 3J). No obvious correlation was observed between the expression of CD64 on monocyte subsets and the proportions of each monocyte subset in HV (data no show).

Expression of CD64 on monocyte subsets correlates with inflammatory markers. Patients with RA frequently have elevated levels of inflammatory markers. To determine the relationship between the expression of CD64 on monocyte subsets and inflammatory markers, inflammatory markers, such as ESR, CRP, white blood cell (WBC), neutrophil count, the percent of neutrophil, IgG, $\mathrm{C} 3$ and $\mathrm{C} 4$, were determined and analyzed for their relationship with the expression of CD64 on $\mathrm{CD} 14^{++} \mathrm{CD} 16-$ monocytes, $\mathrm{CD} 14^{++} \mathrm{CD} 16^{+}$monocytes and $\mathrm{CD} 14^{+} \mathrm{CD} 16^{++}$monocytes in patients with RA. The expression of $\mathrm{CD} 64$ on $\mathrm{CD} 14^{++} \mathrm{CD} 16^{-}$monocytes positively correlated with ESR and CRP in RA patients $(r=0.4853$, $\mathrm{P}=0.0013$, Fig. 4A; $\mathrm{r}=0.4484, \mathrm{P}=0.0061$, Fig. 4B), the expression of $\mathrm{CD} 64$ on $\mathrm{CD} 14^{++} \mathrm{CD} 16^{+}$monocytes positively correlated with ESR and CRP in RA patients ( $\mathrm{r} 0.5128$, $\mathrm{P}=0.0006$, Fig. $4 \mathrm{C} ; \mathrm{r}=0.4721, \mathrm{P}=0.0036$, Fig. 4D), the expression of $\mathrm{CD} 64$ on $\mathrm{CD} 14^{+} \mathrm{CD} 16^{++}$monocytes positively correlated with ESR ( $r=0.3336, P=0.0330$, Fig. 4E), whereas the expression of $\mathrm{CD} 64$ on $\mathrm{CD} 14^{+} \mathrm{CD} 16^{++}$monocytes did not correlate with CRP ( $r=0.1356, P=0.4297$, Fig. 4F). However, no obvious relationship was found between the expression of CD64 on $\mathrm{CD} 14^{++} \mathrm{CD} 16^{-}$monocytes, $\mathrm{CD} 14^{+} \mathrm{CD} 16^{++}$monocytes, $\mathrm{CD} 14^{++} \mathrm{CD} 16^{+}$monocytes and WBC, neutrophil count, the percent of neutrophil, IgG, C3, C4 (data no show).

Expression of CD64 on monocyte subsets correlates with markers of autoimmune response. The hallmark antibodies of RA, such as RF and ACPA, were determined and analyzed for their correlation with the expression of CD64 on monocyte subsets. As shown in Fig. 5, the expression of CD64 on $\mathrm{CD} 14^{++} \mathrm{CD} 16^{-}$monocytes and $\mathrm{CD} 14^{++} \mathrm{CD} 16^{+}$monocytes were significantly increased in patients with positive ACPA and RF respectively ( $\mathrm{P}=0.0460$, Fig. 5A; $\mathrm{P}=0.0035$, Fig. 5B; $\mathrm{P}=0.0416$, Fig. $5 \mathrm{C} ; \mathrm{P}=0.0042$, Fig. 5D). However, no obvious relationship was found between the expression of $\mathrm{CD} 64$ on $\mathrm{CD} 14^{+} \mathrm{CD} 16^{++}$monocytes and ACPA, $\mathrm{RF}(\mathrm{P}=0.6718$, Fig. 5E; $\mathrm{P}=0.8128$, Fig. 5F).

Expression of CD64 on monocyte subsets correlates with disease activity of $R A$. Aforementioned data indicated that the expression of CD64 on monocyte subsets was correlated with markers of inflammation and autoimmune response. 
A
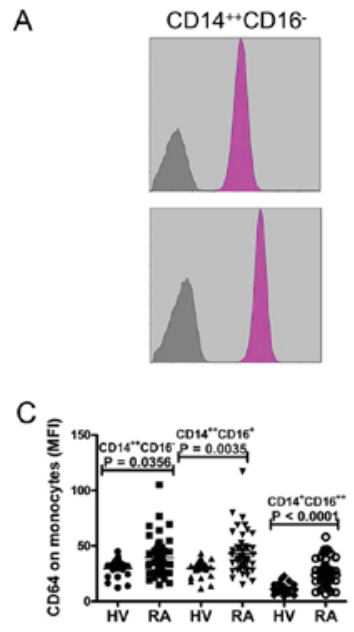

G

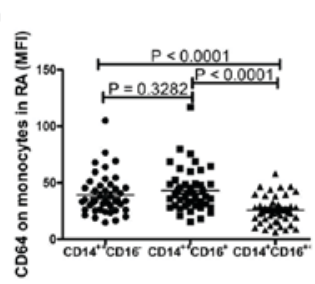

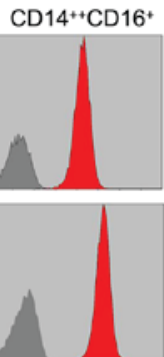

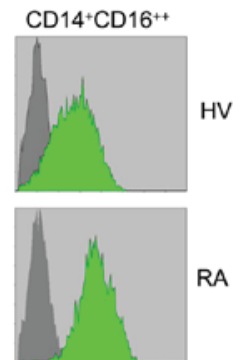

E
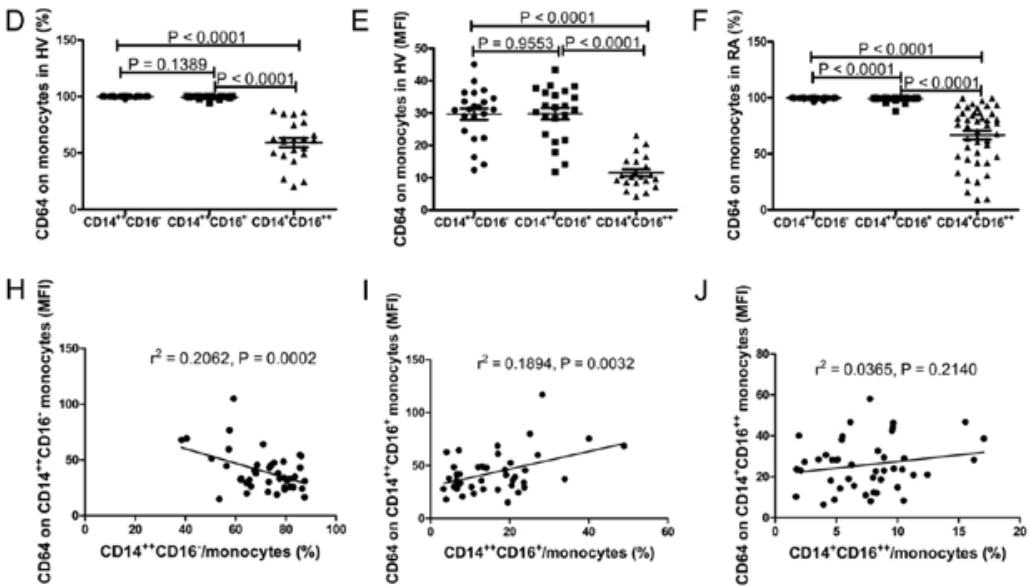

Figure 3. CD64 expression levels are increased in monocyte subsets of RA patients. (A) Flow cytometry analysis of CD64 expression on monocyte subsets from HV and RA patients. CD64-positive cells appear in pink $\left(\mathrm{CD} 14^{++} \mathrm{CD} 16^{-}\right)$, red $\left(\mathrm{CD} 14^{++} \mathrm{CD} 16^{+}\right)$or green $\left(\mathrm{CD} 14^{+} \mathrm{CD} 16^{++}\right)$. Gray histograms represent internal negative controls. Data are cell populations of representative donors. (B) Summary data of the CD64 positive cell frequency on each blood monocyte subset of HV and RA patients. (C) Summary data of the CD64 expression MFI on each blood monocyte subset of HV and RA patients. (D) Summary data of the CD64 positive cell frequency on each blood monocyte subset of HV. (E) Summary data of the CD64 expression (MFI) on each blood monocyte subset of HV. (F) Summary data of the CD64 positive cell frequency on each blood monocyte subset of RA patients. (G) Summary data of the CD64 expression (MFI) on each blood monocyte subset of RA patients. (H) The proportion of $\mathrm{CD} 14^{++} \mathrm{CD} 16$ monocytes negatively correlated with the expression of CD64 on $\mathrm{CD} 4^{++} \mathrm{CD} 16^{-}$monocytes in RA patients. (I) The proportion of $\mathrm{CD} 14^{++} \mathrm{CD} 16^{+}$monocytes positively correlated with the expression of $\mathrm{CD} 64$ on $\mathrm{CD} 14^{++} \mathrm{CD} 16^{+}$ monocytes. (J) No obvious correlation was observed between the expression of CD64 on $\mathrm{CD} 14^{+} \mathrm{CD} 16^{++}$monocytes and the proportions of $\mathrm{CD} 14^{+} \mathrm{CD} 16^{++}$ monocytes. RA, rheumatoid arthritis; HV, health volunteers, MFI, mean fluorescence intensity.
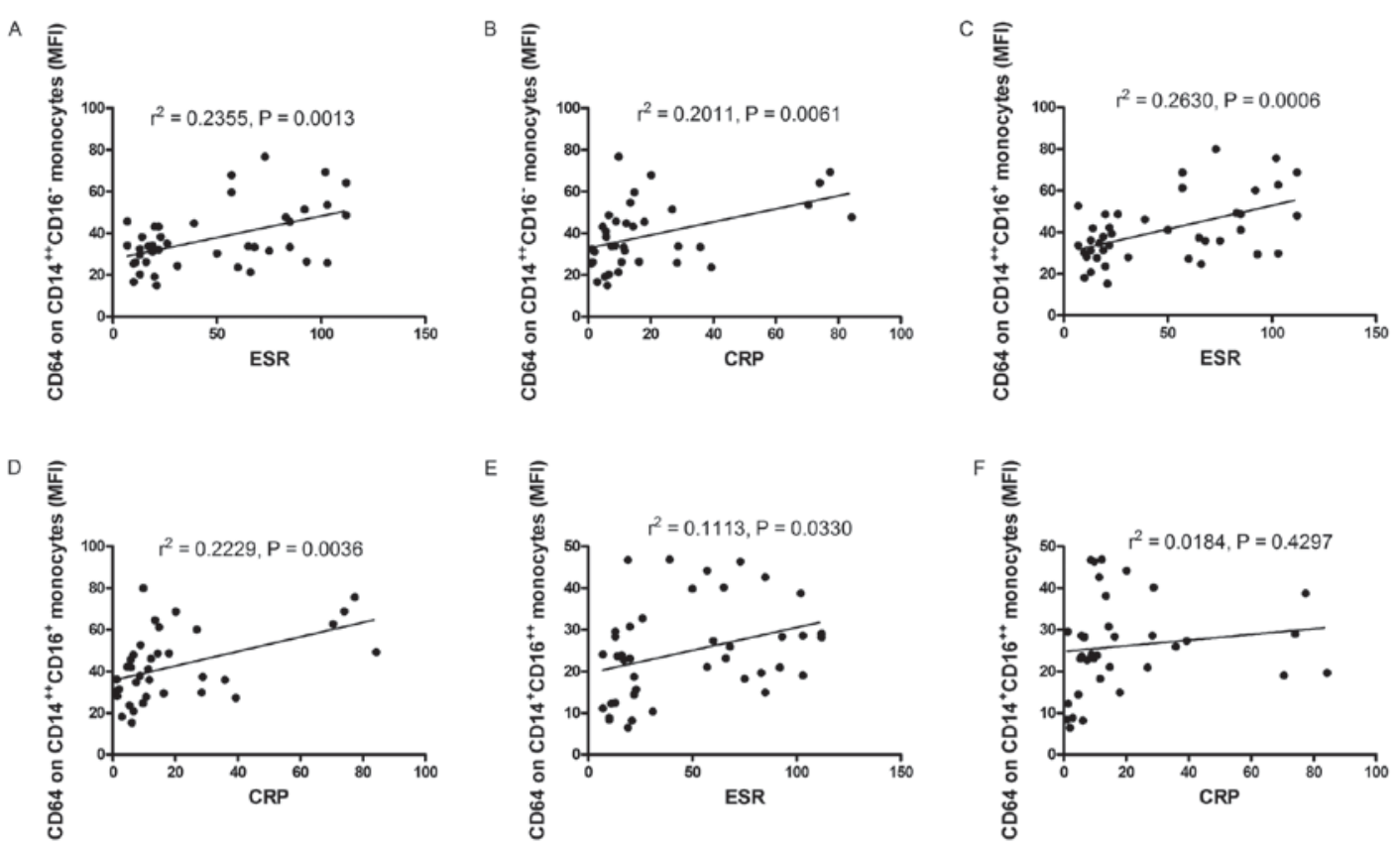

Figure 4. Correlation of the CD64 expression on monocyte subsets with inflammatory markers of RA patients. (A) The expression of CD64 on CD14 ${ }^{++} \mathrm{CD} 16$ monocytes positively correlated with ESR in RA patients. (B) The expression of CD64 on CD14 ${ }^{++} \mathrm{CD} 16^{-}$monocytes positively correlated with CRP in RA patients. (C) The expression of CD64 on CD14 ${ }^{++} \mathrm{CD} 16^{+}$monocytes positively correlated with ESR in RA patients. (D) The expression of $\mathrm{CD} 64$ on $\mathrm{CD} 14^{++} \mathrm{CD} 16^{+}$ monocytes positively correlated with CRP in RA patients. (E) The expression of CD64 on CD14 ${ }^{+} \mathrm{CD}_{16} 6^{++}$monocytes positively correlated with ESR in RA patients. (F) The expression of CD64 on CD14 ${ }^{+} \mathrm{CD} 16^{++}$monocytes did not correlate with CRP in RA patients. RA, rheumatoid arthritis; ESR, erythrocyte sedimentation rate; $\mathrm{CRP}, \mathrm{C}$-reactive protein. 



Figure 5. Correlation of the CD64 expression on monocyte subsets with autoantibody of RA patients. (A) The expression of CD64 on CD14 ${ }^{++}$CD16- monocytes was significantly increased in RA patients with positive ACPA. (B) The expression of CD64 on CD14 ${ }^{++} \mathrm{CD} 16$ monocytes was significantly increased in RA patients with positive RF. (C) The expression of $\mathrm{CD} 64$ on $\mathrm{CD} 14^{++} \mathrm{CD} 16^{+}$monocytes were significantly increased in RA patients with positive ACPA. (D) The expression of $\mathrm{CD} 64$ on $\mathrm{CD} 14^{++} \mathrm{CD} 16^{+}$monocytes were significantly increased in RA patients with positive RF. (E) No obvious relationship was found between the expression of CD64 on $\mathrm{CD} 14^{+} \mathrm{CD} 16^{++}$monocytes and ACPA. (F) No obvious relationship was found between the expression of $\mathrm{CD} 64$ on $\mathrm{CD} 14^{+} \mathrm{CD} 16^{++}$ monocytes and RF. RA, rheumatoid arthritis; ACPA, anti-citrullinated protein antibodies; RF, rheumatoid factor.

Thus, the correlation between the expression of CD64 on monocyte subsets and disease activity were investigated. Data showed that both the expression of CD64 on $\mathrm{CD} 14^{++} \mathrm{CD} 16$ monocytes and $\mathrm{CD} 14^{++} \mathrm{CD} 16^{+}$monocytes were positively correlated with DAS28 score $(\mathrm{r}=0.3506, \mathrm{P}=0.0212 ; \mathrm{r}=0.3208$, $\mathrm{P}=0.0360$ ) (Fig. 6A and B), while the expression of CD64 on $\mathrm{CD} 14^{+} \mathrm{CD} 16^{++}$monocytes did not correlate with DAS28 score ( $r=0.2587, \mathrm{P}=0.0938$; Fig. 6C).

Subsequently, we compared the CD64 expression on monocyte subsets between patients with new-onset and re-visiting RA. Data showed that the expression of CD64 on monocytes subsets tends to be elevated in patients with new-onset RA, but a significant difference was not reached (P>0.0500; Fig. 6D).

Association between the expression of CD64 on monocyte subsets and serum cytokine concentration. Among the three serum inflammatory cytokines, levels of IL-6 and IL-8 were significantly higher in patients with RA than in HV ( $\mathrm{P}=0.0011$, Fig. 7A; $\mathrm{P}=0.0387$, Fig. 7B), no significant difference was observed in the levels of IL-10 between patients with RA and HV ( $\mathrm{P}=0.8994$; Fig. 7C). To determine whether increased levels of CD64 on monocyte subsets play a role in the secretion of serum cytokines (IL-6 and IL-8), RA patients were divided into two groups according to their CD64 levels (average of MFI) on monocytes subsets: RA high $\left(\mathrm{CD} 64\right.$ on $\mathrm{CD} 4^{++} \mathrm{CD}^{-} 6^{-} 39.32, \mathrm{CD} 4$ on $\mathrm{CD} 14^{++} \mathrm{CD} 16^{+}$ $>43.19, \mathrm{CD} 64$ on $\left.\mathrm{CD}_{14}{ }^{+} \mathrm{CD} 16^{++}>25.87\right)$ and $\mathrm{RA}^{\text {low }}(\mathrm{CD} 64$ on CD14 ${ }^{++} \mathrm{CD} 166^{-}<39.32$, CD64 on $\mathrm{CD} 14^{++} \mathrm{CD} 16^{+}<43.19$, CD64 on $\left.\mathrm{CD} 14^{+} \mathrm{CD} 16^{++}<25.87\right)$. RA patients with high levels of CD64 on $\mathrm{CD} 14^{++} \mathrm{CD} 16^{+}$monocytes exhibited significantly higher levels of IL- 6 compared with RA patients with low levels of $\mathrm{CD} 64$ on $\mathrm{CD} 14^{++} \mathrm{CD} 16^{+}$monocytes $(\mathrm{P}=0.0131$;
Fig. 7D). No significant difference was observed in the levels of IL- 6 between RA patients with high levels of CD64 on $\mathrm{CD} 14^{++} \mathrm{CD} 16^{-}$or $\mathrm{CD} 14^{+} \mathrm{CD} 16^{++}$monocytes and low levels of CD64 on $\mathrm{CD} 14^{++} \mathrm{CD} 16^{-}$or $\mathrm{CD} 14^{+} \mathrm{CD} 16^{++}$monocytes ( $P>0.05$; Fig. 7D). And, no significant difference was observed in the levels of IL-8 between RA patients with high levels of CD64 on each monocyte subset and low levels of CD64 on each monocyte subset $(\mathrm{P}>0.05$; Fig. $7 \mathrm{E})$. These results indicate that increased levels of CD64 on $\mathrm{CD} 14^{++} \mathrm{CD} 16^{+}$monocytes in RA patients are associated with increased secretion of IL-6.

\section{Discussion}

Monocytes are a heterogeneous cell population composed of classical monocytes $\left(\mathrm{CD} 14^{++} \mathrm{CD} 16^{-}\right)$, intermediate monocytes $\left(\mathrm{CD} 14^{++} \mathrm{CD} 16^{+}\right)$and nonclassical monocytes $\left(\mathrm{CD} 14^{+} \mathrm{CD} 16^{++}\right)$. The three subsets of monocytes perform different functions. The classical subset is rapidly recruited to the sites of inflammation and appears to act as phagocytic scavenger cells and regulators of inflammation $(22,23)$. The intermediate monocytes play a proinflammatory role, being increased in blood from patients with acute inflammation $(24,25)$. The nonclassical monocytes are often referred to as patrolling monocytes (26). Previous researchs have reported monocytes play a important role in the progression of RA. The onset and severity of RA might also be due to the seasonality of monocyte subsets was aberrant.

Although an increase in $\mathrm{CD} 14^{++} \mathrm{CD} 16^{+}$monocytes and an decreased in $\mathrm{CD}_{14}{ }^{++} \mathrm{CD} 16^{-}$monocytes in patients with $\mathrm{RA}$ have been reported, the increase in $\mathrm{CD} 14^{+} \mathrm{CD} 16^{++}$monocytes remained controversial $(27,28)$. In consistent with the report of Patricia Lacerte (28), this study demonstrate that circulating 



D

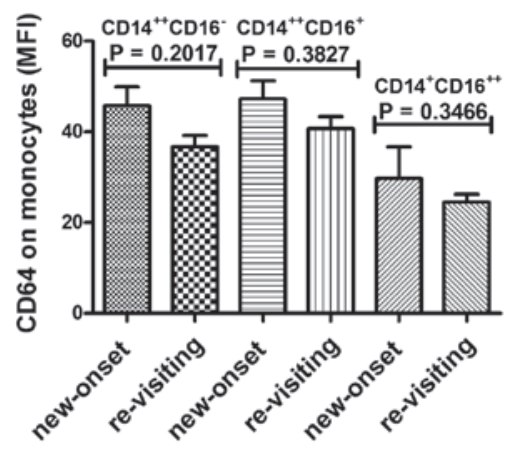

Figure 6. Correlation of the CD64 expression on monocyte subsets with disease activity of RA patients. (A) The expression of CD64 on CD14 ${ }^{++} \mathrm{CD} 16^{-}$monocytes was positively correlated with DAS28 score in RA patients. (B) The expression of CD64 on CD14 $4^{++} \mathrm{CD} 16^{+}$monocytes was positively correlated with DAS28 score in RA patients. (C) The expression of $\mathrm{CD} 64$ on $\mathrm{CD} 14^{+} \mathrm{CD} 16^{++}$monocytes did not correlate with DAS28 score in RA patients. (D) The expression of CD64 on monocytes subsets tends to be elevated in patients with new-onset RA patients, but a significant difference was not reached. RA, rheumatoid arthritis.
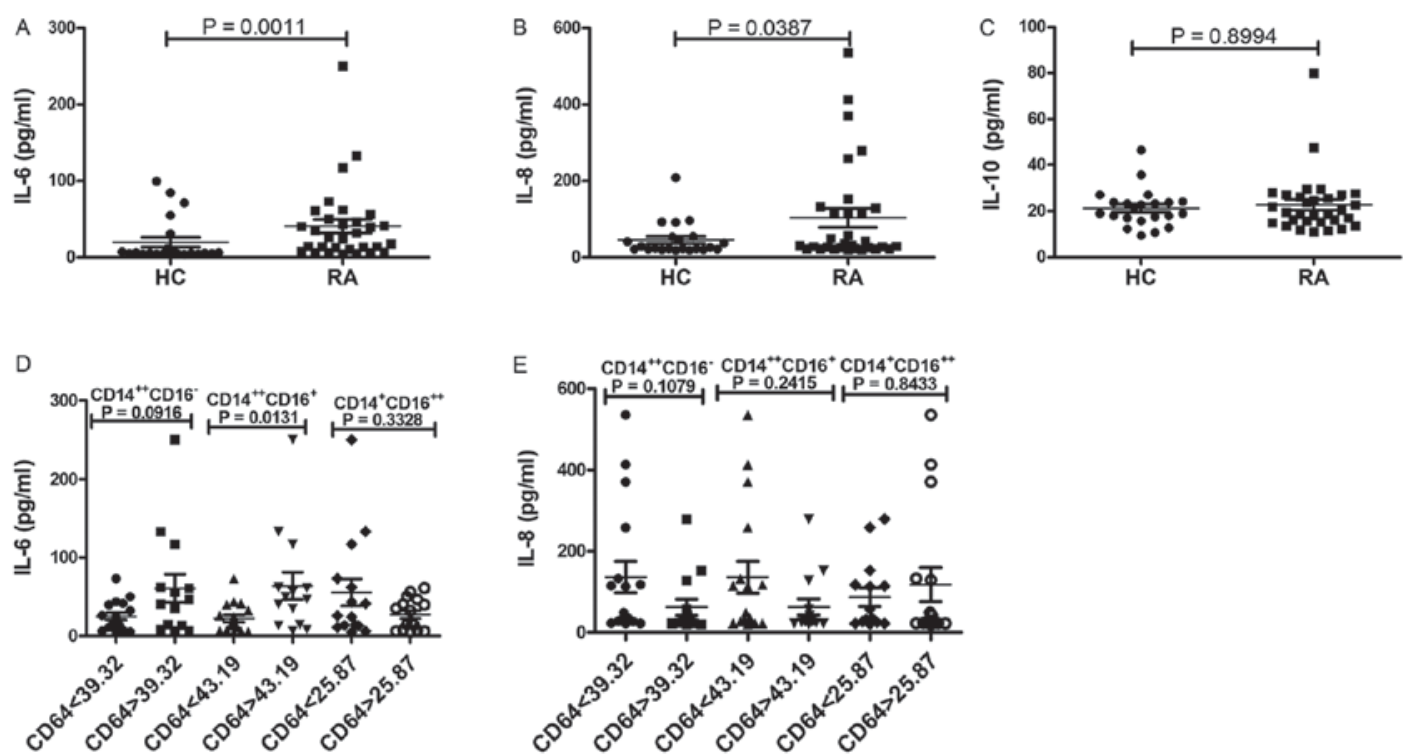

Figure 7. Correlation of the CD64 expression on monocyte subsets with serum cytokines (IL-6 and IL-8) of RA patients. (A) The levels of IL-6 was significantly increased in patients with RA compared with HV. (B) The levels of IL-8 was significantly increased in patients with RA compared with HV. (C) No significant difference was observed in the levels of IL-10 between patients with RA and HV. (D) Summary data of the levels of IL-6 between RA patients with high levels of CD64 on monocyte subsets and low levels of CD64 on monocyte subsets. (E) Summary data of the levels of IL-8 between RA patients with high levels of CD64 on monocyte subsets and low levels of CD64 on monocyte subsets. RA, rheumatoid arthritis; HV, health volunteers.

$\mathrm{CD} 14^{++} \mathrm{CD} 16^{+}$and $\mathrm{CD} 14^{+} \mathrm{CD} 16^{++}$monocytes are increased, while circulating $\mathrm{CD}_{14}^{++} \mathrm{CD} 16^{-}$monocytes are decreased in patients with RA. The reasons for these outcomes are probably due to differences in the disease duration and ongoing treatments. 
An assessment of the expression of the characteristic phenotypic markers CD40, CD64, CD163, CD206, HLA-DR, CD80 and CD86 helped to characterize further the monocyte response in patients with RA. In consistent with the results of other researches $(16,29)$, we found that the expression of CD64 on monocytes was significantly elevated in RA patients compared to HV, no changes of other markers between patients with RA and HV. The increased expression of CD64 on monocytes in patients with active RA may suggest the progression of the disease (16), and may also reflect the activation of the monocytes. Although an increase in CD64 on monocytes subsets in patients with RA has been reported (30), the possibility of correlation between the expression of CD64 on each monocytes subset and disease activity in patients with RA has not yet been investigated. Our results support previous observations (30) and show that the expression of CD64 on $\mathrm{CD} 14^{++} \mathrm{CD} 16-$ monocytes and $\mathrm{CD} 14^{++} \mathrm{CD} 16^{+}$monocytes were significantly elevated compared to $\mathrm{CD} 14^{+} \mathrm{CD} 16^{++}$monocytes in RA patients, and the expression of $\mathrm{CD} 64$ on $\mathrm{CD} 14^{++} \mathrm{CD} 16$ monocytes and $\mathrm{CD} 14^{++} \mathrm{CD} 16^{+}$monocytes were positively correlated with DAS28 score.

Little is known about the possibility of correlation between the expression of CD64 on each monocyte subset and the proportion of each monocyte subset in patients with $\mathrm{RA}$. We found that the proportion of $\mathrm{CD} 14^{++} \mathrm{CD} 16^{+}$monocytes positively correlated with the expression of CD64 on $\mathrm{CD} 14^{++} \mathrm{CD} 16^{+}$monocytes in RA patients, whereas the proportion of $\mathrm{CD} 14^{++} \mathrm{CD} 16^{-}$monocytes negatively correlated with the expression of $\mathrm{CD} 64$ on $\mathrm{CD} 14^{++} \mathrm{CD} 16^{-}$monocytes. The reasons for the results are probably due to the facts that the proportion of intermediate monocytes positively correlated with the disease activity of RA, whereas the proportion of classical monocytes negatively correlated (27) and our results showed that the expression of $\mathrm{CD} 64$ on $\mathrm{CD} 14^{++} \mathrm{CD} 16^{-}$monocytes and $\mathrm{CD} 14^{++} \mathrm{CD} 16^{+}$monocytes were positively correlated with DAS28 score.

It is well-known that RA is a autoimmune disease characterized by the production of autoantibodies including RF, ACPA and autoimmune response is a kind of chronic inflammation against self antigens. In this study, the inflammatory markers, DAS28, the hallmark antibodies of RA including RF and ACPA were first determined and analyzed for their relation with the expression of CD64 on monocyte subsets. Our results showed that the expression of CD64 on $\mathrm{CD} 14^{++} \mathrm{CD} 16^{-}$and $\mathrm{CD} 14^{++} \mathrm{CD} 16^{+}$monocytes were positively related with ESR, CRP and DAS28, whereas the expression of CD64 on $\mathrm{CD} 14^{+} \mathrm{CD} 16^{++}$monocytes did not correlate with CRP and DAS28. In addition, we found the expression of $\mathrm{CD} 64$ on $\mathrm{CD} 14^{++} \mathrm{CD} 16-$ monocytes and $\mathrm{CD} 14^{++} \mathrm{CD} 16^{+}$monocytes were significantly increased in patients with positive RF and ACPA respectively, whereas no obvious relationship was found between the expression of $\mathrm{CD} 64$ on $\mathrm{CD} 14^{++} \mathrm{CD} 16^{+}$ monocytes and RF, ACPA. This may be that (1) $\mathrm{CD} 14^{++} \mathrm{CD} 16$ monocytes and $\mathrm{CD} 14^{++} \mathrm{CD} 16^{+}$monocytes appears to act as regulators of inflammation, whereas $\mathrm{CD} 14^{+} \mathrm{CD} 16^{++}$monocytes often referred to as patrolling monocytes (22-26); (2) CD64 is a high-affinity activating receptor that can bind IgG and CRP and stimulate inflammatory processes $(16,31,32)$.

In consistent with previous study (27), we showed here that levels of IL-6 and IL-8 at baseline were significantly higher in patients with RA than in HV. In addition, we observed that RA patients with high levels of CD64 on $\mathrm{CD} 14^{++} \mathrm{CD} 16^{+}$monocytes exhibited significantly higher levels of IL-6 compared with the RA patients with low levels of $\mathrm{CD} 64$ on $\mathrm{CD} 14^{++} \mathrm{CD} 16^{+}$ monocytes. These results suggested that the levels of CD64 on $\mathrm{CD} 14^{++} \mathrm{CD} 16^{+}$monocytes is indeed linked to the secretion high concentrations of proinflammatory cytokines.

However, there are some limitations in the present study. First is the relatively small sample size, especially the sample of new-onset RA; these data may be confirmed in large-scale studies. Second, we did not show that each monocyte subset are directly associated with inflammatory cytokines in RA in vitro. Third, No function study and experiments about the mechanism of CD64 have been done in this study. The molecular mechanisms underlying CD64 functions in RA still require further investigation.

In conclusions, results presented in this study demonstrate that blood monocyte subsets isolated from patients with RA have high levels of CD64 and the levels of CD64 on $\mathrm{CD} 14^{++} \mathrm{CD} 16^{-}$and $\mathrm{CD} 14^{++} \mathrm{CD} 16^{+}$monocytes correlates with the disease activity of RA. In addition, the levels of CD64 on $\mathrm{CD} 14^{++} \mathrm{CD} 16^{+}$monocytes is linked to the high secretion level of proinflammatory cytokines.

\section{Acknowledgements}

The authors would like to acknowledge the help from Dr Rui Wu from the Department of Rheumatology, The First Affiliated Hospital of Nanchang University, Nanchang, Jiangxi, China.

\section{Funding}

The present study was supported by the National Natural Science Foundation of China (grant no. 81360459), Jiangxi Provincial Natural Science Foundation of China (grant nos. 20151BAB215031 and 20171BAB205113), the Science and Technology Project of Health and Family Planning Commission of Jiangxi Province of China (grant no. 20165094), the Science and Technology Plan Project of the Education Department of Jiangxi Province (grant no. GJ170008) and the Foundation for Distinguished Young Scientists of the Jiangxi Province of China (grant no. 20171BCB23087).

\section{Availability of data and materials}

The datasets used and/or analyzed during the current study are available from the corresponding author on reasonable request.

\section{Authors' contributions}

QL participated in designing the study, performed statistical analyses and drafted the manuscript. PCX participated in the design of the study and helped to revise the manuscript. XL performed flow cytometry analysis and drafted the manuscript. ZD performed statistical analyses and drafted the manuscript. CQ performed data acquisition of markers of autoimmune response, performed statistical analyses and drafted the manuscript. RGS carried out data acquisition of markers of inflammation, performed statistical analyses and drafted the manuscript. JQX performed data acquisition of 
disease activity and severity, performed statistical analyses, and drafted the manuscript. YG carried out the experiments on the expression of cytokines and drafted the manuscript. $\mathrm{ZKH}$ and JML conceived of the study, participated in its design and coordination, and helped to draft the manuscript. All authors read and approved the final manuscript.

\section{Ethics approval and consent to participate}

The study was approved by the Ethics Committee of the First Affiliated Hospital of Nanchang University (019) and was carried out in compliance with the Helsinki Declaration. Written informed consent was obtained from all participants before they entered the study.

\section{Patient consent for publication}

Not applicable.

\section{Competing interests}

The authors declare that they have no competing interests.

\section{References}

1. Li X, Yuan FL, Lu WG, Zhao YQ, Li CW, Li JP and Xu RS: The role of interleukin-17 in mediating joint destruction in rheumatoid arthritis. Biochem Biophys Res Commun 397: 131-135, 2010

2. Yuan FL, Li X, Lu WG, Li CW, Xu RS and Dong J: IL-33: A promising therapeutic target for rheumatoid arthritis? Expert Opin Ther Targets 15: 529-534, 2011.

3. McInnes IB and Schett G: The pathogenesis of rheumatoid arthritis. N Engl J Med 365: 2205-2219, 2011.

4. Cascão R, Rosário HS, Souto-Carneiro MM and Fonseca JE: Neutrophils in rheumatoid arthritis: More than simple final effectors. Autoimmun Rev 9: 531-535, 2010.

5. Shi C and Pamer EG: Monocyte recruitment during infection and inflammation. Nat Rev Immunol 11: 762-774, 2011.

6. Wong KL, Yeap WH, Tai JJ, Ong SM, Dang TM and Wong SC: The three human monocyte subsets: Implications for health and disease. Immunol Res 53: 41-57, 2012.

7. Ziegler-Heitbrock L: The CD14+ CD16+ blood monocytes: Their role in infection and inflammation. J Leukoc Biol 81: 584-592, 2007.

8. Skrzeczyńska-Moncznik J, Bzowska M, Loseke S, Grage-Griebenow E, Zembala M and Pryjma J: Peripheral blood CD14high CD16+ monocytes are main producers of IL-10. Scand J Immunol 67: 152-159, 2008.

9. Rossol M, Kraus S, Pierer M, Baerwald C and Wagner U. The CD14 (bright) CD16+ monocyte subset is expanded in rheumatoid arthritis and promotes expansion of the Th17 cell population. Arthritis Rheum 64: 671-677, 2012.

10. Tsukamoto M, Seta N, Yoshimoto K, Suzuki K, Yamaoka K and Takeuchi T: CD14brightCD16+ intermediate monocytes are induced by interleukin-10 and positively correlate with disease activity in rheumatoid arthritis. Arthritis Res Ther 19: 28, 2017.

11. Nimmerjahn F and Ravetch JV: Fc $\gamma$ receptors as regulators of immune responses. Nat Rev Immunol 8: 34-47, 2008.

12. Amigorena $S$ and Bonnerot $\mathrm{C}$ : Fc receptor signalling and trafficking: A connection for antigen processing. Immunol Rev 172: 279-284, 1999.

13. García-García E and Rosales C: Signal transduction during Fc receptor-mediated phagocytosis. J Leukoc Biol 72: 1092-1108, 2002.

14. Magnusson SE, Engström M, Jacob U, Ulfgren AK and Kleinau S: High synovial expression of the inhibitory FcgammaRIIb in rheumatoid arthritis. Arthritis Res Ther 9: R51, 2007.
15. van Vuuren AJ, van Roon JA, Walraven V, Stuij I, Harmsen MC, McLaughlin PM, van de Winkel JG and Thepen T: CD64-directed immunotoxin inhibits arthritis in a novel CD64 transgenic rat model. J Immunol 176: 5833-5838, 2006.

16. Matt P, Lindqvist U and Kleinau S: Elevated membrane and soluble CD64: A novel marker reflecting altered fc $\gamma \mathrm{r}$ function and disease in early rheumatoid arthritis that can be regulated by anti-rheumatic treatment. PLoS One 10: e0137474, 2015.

17. Laurent L, Clavel C, Lemaire O, Anquetil F, Cornillet M, Zabraniecki L, Nogueira L, Fournié B, Serre G and Sebbag M: $\mathrm{Fc} \gamma$ receptor profile of monocytes and macrophages from rheumatoid arthritis patients and their response to immune complexes formed with autoantibodies to citrullinated proteins. Ann Rheum Dis 70: 1052-1059, 2011

18. Hepburn AL, Mason JC and Davies KA: Expression of Fc $\gamma$ and complement receptors on peripheral blood monocytes in systemic lupus erythematosus and rheumatoid arthritis. Rheumatology (Oxford) 43: 547-554, 2004

19. Arnett FC, Edworthy SM, Bloch DA, McShane DJ, Fries JF, Cooper NS, Healey LA, Kaplan SR, Liang MH,Luthra HS, et al: The american rheumatism assocaition 1987 revised criteria for the classification of rheumatoid arthritis. Arthritis Rheum 31: 315-324, 1988.

20. Wang J, Shan Y, Jiang Z, Feng J, Li C, Ma L and Jiang Y: High frequencies of activated $\mathrm{B}$ cells and $\mathrm{T}$ follicular helper cells are correlated with disease activity in patients with new-onset rheumatoid arthritis. Clin Exp Immunol 174: 212-220, 2013.

21. Prevoo ML, van't Hof MA, Kuper HH, van Leeuwen MA, van de Putte LB and van Riel PL: Modified disease activity scores that include twenty-eight-joint counts. Development and validation in a prospective longitudinal study of patients with rheumatoid arthritis. Arthritis Rheum 38: 44-48, 1995.

22. Mehta NN and Reilly MP: Monocyte mayhem: Do subtypes modulate distinct atherosclerosis phenotypes? Circ Cardiovasc Genet 5: 7-9, 2012

23. Mobley JL, Leininger M, Madore S, Baginski TJ and Renkiewicz R: Genetic evidence of a functional monocyte dichotomy. Inflammation 30: 189-197, 2007.

24. Auffray C, Sieweke MH and Geissmann F: Blood monocytes: Development, heterogeneity and relationship with dendritic cells. Annu Rev Immunol 27: 669-692, 2009.

25. Belge KU, Dayyani F, Horelt A, Siedlar M, Frankenberger M, Frankenberger B, Espevik T and Ziegler-Heitbrock L: The proinflammatory CD14 + CD16 + DR++ monocytes are a major source of TNF. J Immunol 168: 3536-3542, 2002.

26. Cros J, Cagnard N, Woollard K, Patey N, Zhang SY, Senechal B, Puel A, Biswas SK, Moshous D, Picard C, et al: Human CD14dim monocytes patrol and sense nucleic acids and viruses via TLR7 and TLR8 receptors. Immunity 33: 375-386, 2010.

27. Tsukamoto M, Seta N, Yoshimoto K, Suzuki K, Yamaoka K and Takeuchi T: CD14brightCD16+intermediate monocytes are induced by interleukin-10 and positively correlate with disease activity in rheumatoid arthritis. Arthritis Res Ther 19: 28, 2017.

28. Lacerte P, Brunet A, Egarnes B, Duchêne B, Brown JP and Gosselin J: Overexpression of TLR2 and TLR9 on monocyte subsets of active rheumatoid arthritis patients contributes to enhance responsiveness to TLR agonists. Arthritis Res Ther 18: 10, 2016.

29. Wijngaarden S, van Roon JA, Bijlsma JW, van de Winkel JG and Lafeber FP: Fcgamma receptor expression levels on monocytes are elevated in rheumatoid arthritis patients with high erythrocyte sedimentation rate who do not use anti-rheumatic drugs. Rheumatology (Oxford) 42: 681-688, 2003.

30. Rossol M, Kraus S, Pierer M, Baerwald C and Wagner U: The CD14brightCD16 monocyte subset is expanded in rheumatoid arthritis and promotes expansion of the th17 cell population. Arthritis Rheum 64: 671-677, 2012.

31. Bruhns P, Iannascoli B, England P, Mancardi DA, Fernandez N, Jorieux S and Daëron M: Specificity and affinity of human Fcgamma receptors and their polymorhic variants for IgG subclasse. Blood 113: 3716-3725, 2009.

32. Lu J, Marjon KD, Marnell LL, Wang R, Mold C, Du Clos TW and Sun P: Recognition and functional activation of the human IgA receptor (FcaRI) by C-reactive protein. Proc Natl Acad Sci USA 108: 4974-4979, 2011. 